\title{
O DESENVOLVIMENTO DO PADRÃO DE VOICE ONSET TIME DAS OCLUSIVAS SURDAS INICIAIS DO INGLÊS POR APRENDIZES SOTEROPOLITANOS: EFEITOS DA INSTRUÇÃO EXPLÍCITA
}

\author{
Felipe Flores Kupske ${ }^{1^{*}}$ \\ Michele Santos de Oliveira ${ }^{1 * *}$ \\ ${ }^{1}$ Universidade Federal da Bahia, Salvador, Bahia, Brasil
}

\begin{abstract}
Resumo
Neste trabalho, investigamos o efeito da instrução explícita de pronúncia no desenvolvimento do padrão de Voice Onset Time (VOT) das oclusivas surdas iniciais do inglês como língua não nativa por aprendizes soteropolitanos. O estudo contou com três coletas de dados, um pré-teste, um pós-teste imediato e um pós-teste postergado, de 16 aprendizes soteropolitanos, divididos em grupos controle e experimental, e com uma sessão de instrução sobre a produção das oclusivas surdas iniciais do inglês. Análises acústicas do VOT são reportadas. Os resultados revelaram que o grupo controle, que não recebeu instrução, não produziu o padrão de VOT esperado para o inglês em nenhuma das coletas. Por outro lado, no grupo experimental, que recebeu instrução explícita, pudemos notar um aumento considerável na duração das oclusivas da L2. Nossos dados revelam efeitos positivos da instrução explícita de pronúncia para o desenvolvimento do VOT da L2 e vão ao encontro de Sancier e Fowler (1997) e Kupske (2016), os quais afirmam que brasileiros são capazes de atingir produções estatisticamente próximas ao esperado para o inglês, ao menos no que concerne ao VOT.

Palavras-chave: VOT; Oclusivas Surdas Iniciais; Instrução Explícita.
\end{abstract}

\footnotetext{
Professor permanente do Programa de Pós-Graduação em Língua e Cultura (PPGLinC) da Universidade Federal da Bahia (UFBA). Líder do Laboratório de Ciências da Fala (LAFALA-UFBA). Doutor em Letras pela Universidade Federal do Rio Grande do Sul (UFRGS). Seu e-mail é kupske@gmail.com. ORCID 0000-00020616-612X.

" Bacharel em Letras pela Universidade Federal da Bahia (UFBA). Membro do Laboratório de Ciências da Fala (LAFALA-UFBA). Seu e-mail é micheleolliveira96@gmail.com. ORCID: 0000-0002-2757-8916.
} 


\title{
THE DEVELOPMENT OF THE VOICE ONSET TIME PATTERN OF INITIAL VOICELESS STOPS OF ENGLISH BY BRAZILIAN LEARNERS FROM SALVADOR, BRAZIL: EFFECTS OF EXPLICIT INSTRUCTION
}

\begin{abstract}
In this work, we investigate the effect of explicit pronunciation instruction on the development of the Voice Onset Time (VOT) pattern of initial voiceless English stops by learners from Salvador, Brazil. The study included three data collections, a pre-test, an immediate post-test and a postponed post-test, from 16 learners, divided into control and experimental groups, and with an explicit instruction session on the production of the English stops. Acoustic analyses of VOT are reported. The results revealed that the control group, which received no instruction, did not produce the expected VOT pattern for English at any time of the study. On the other hand, in the experimental group, which received explicit instruction, we could perceive a considerable increase in the duration of the L2 stops. Our data reveal positive effects of explicit pronunciation instruction for the development of English VOT and are in line with Sancier and Fowler (1997) and Kupske (2016), who state that BP speakers are able to achieve productions that are statistically close to what is expected for English, at least as far as VOT is concerned.

Keywords: VOT; Word-initial Plosives; Explicit Instruction.
\end{abstract}




\section{Introdução}

Para Mauranen e Ranta (2009), o inglês é um dos símbolos de nosso tempo. No atual mundo inter e transcultural, o inglês surge como uma língua de contato usada por falantes de diferentes origens linguísticas e culturais, funcionando como uma "língua franca" global (SEIDLHOFER, 2011). Como uma das consequências da expansão da língua inglesa como "língua franca", as últimas décadas têm visto um crescente interesse (e necessidade) na investigação sobre o construto da inteligibilidade (JENKINS, 2000; BECKER, 2014; ALVES; KAMPFF, 2019), alimentando, por exemplo, a pesquisa nas áreas da interfonologia e da instrução de pronúncia, que, para Thomson e Derwing (2014, p. 2), deveria se preocupar principalmente em auxiliar os aprendizes de línguas não nativas a se tornarem mais compreensíveis.

No que concerne à inteligibilidade ${ }^{1}$ oral do inglês, foco deste trabalho, uma característica acústica que tem sido considerada importante é a extensão do Voice Onset Time (VOT), definido como o intervalo de tempo entre a soltura de uma consoante oclusiva e o início da pulsão glotal (anterior ou posterior à soltura) (LISKER; ABRAMSON, 1964). Pesquisas como a de Alves e Kampff (2019), por exemplo, indicam que o VOT é a principal pista acústica do inglês para o estabelecimento do contraste entre oclusivas iniciais surdas e sonoras.

Embora não exista uma medida absoluta para valores de VOT para cada oclusiva surda, no português brasileiro (PB), segundo Istre (1980), os valores variam em torno de $38 \mathrm{~ms}$ para /k/, $18 \mathrm{~ms}$ para /t/ e $12 \mathrm{~ms}$ para /p/. Muito embora alguns estudos (REIS; NOBRE-OLIVEIRA, 2007; SCHWARTZHAUPT, 2013; ALVES; ZIMMER, 2015) apontem valores mais altos para a oclusiva velar, que podem chegar a $63.90 \mathrm{~ms}$ no $\mathrm{PB}$ falado em algumas regiões do Brasil, as oclusivas surdas do $\mathrm{PB}$ são tipicamente classificadas como tendo um VOT próximo de zero, sem pré-vozeamento e sem aspiração (VOT curto, short lag) (CRISTÓFARO-SILVA et al., 2019). Alguns autores, como Reis e Nobre-Oliveira (2007), consideram que as oclusivas surdas tendem a ser produzidas com um VOT positivo, mas curto em qualquer contexto.

Em inglês, por outro lado, o VOT para esse grupo de consoantes tende a ser longo em posição inicial de palavra e em sílabas tônicas, VOT positivo (VOT longo, long lag). Toribio et al (2005) e Kupske (2016) apontam que os valores típicos do inglês estão em torno dos $80 \mathrm{~ms}$ para /k/, $70 \mathrm{~ms}$ para /t/, e $55 \mathrm{~ms}$ para /p/. Em adição, na língua inglesa, oclusivas produzidas com um VOT positivo, aspiradas (long lag), são identificadas como consoantes surdas por seus falantes nativos (SCHWARTZHAUPT; ALVES; FONTES, 2015; ALVES; KAMPFF, 2019). No entanto, oclusivas com padrão de VOT Zero ou com padrão de VOT Negativo, apresentando vibração de pregas vocais antes da soltura, seriam identificadas pelos falantes nativos do inglês como consoantes sonoras (ALVES; KAMPFF, 2019). Em outras palavras, para o inglês, seria a presença ou ausência de aspiração, de uma maior duração, que levaria à discriminação entre oclusivas surdas (/p, t, k/) e sonoras (/b, d, g/). Contudo, devemos levar em consideração que o VOT não 
corresponde a uma pista acústica para distinções de sonoridade entre o grupo das oclusivas em todas as línguas naturais, a exemplo do PB, para o qual as oclusivas surdas são produzidas com um VOT curto, sem aspiração, como já mencionado. Em suma, o PB e o inglês pertencem a grupos de padrões de VOT distintos.

Ao levarmos em consideração (i) que há transferência fonético-fonológica entre língua materna (L1) e segunda língua ${ }^{2}$ (L2) no desenvolvimento de aprendizes, pela qual padrões linguísticos mais bem estabelecidos se transferem para padrões menos robustos (KUPSKE; ALVES, 2017), e (ii) que a inteligibilidade é um fator decisivo para a manutenção do inglês como "língua franca" (ILF), que tem como principal objetivo funcionar como uma língua-ponte entre falantes que não compartilham a mesma língua e/ou cultura maternas, precisamos considerar um ensino de inglês-L2, como sinalizam Alves e Kampff (2019), que desenvolva no aprendiz a realização do VOT apropriado, tanto no âmbito de produção como de percepção da L2. Em adição, para esses autores, uma rotina baseada apenas no treinamento perceptual, embora contribua modestamente para a melhora da percepção das oclusivas no inglês-L2, não reflete em ganhos generalizáveis quanto à produção (ALVES; KAMPFF, 2019). Neste trabalho, defendemos, então, um modelo de ensino comunicativo de pronúncia que foque não apenas na percepção, mas também na produção dos sons da L2, como proposto por Celce-Murcia et al (1996) e Celce-Murcia et al (2010), para o desenvolvimento do VOT do inglês-L2.

Advogamos o modelo de Celce-Murcia et al (1996) e Celce-Murcia et al (2010), pois resultados positivos na produção da L2 são geralmente encontrados quando a instrução é acompanhada de feedback corretivo (SAITO, 2012), de instrução explícita (GORDON; DARCY, 2016; THOMSON; DERWING, 2015), e de conscientização das diferenças interlinguísticas entre L1 e L2 (ALVES; MAGRO, 2011; SILVEIRA; ALVES, 2009), características presentes entre os cinco passos propostos pelo modelo. Os passos são estes: (i) descrição e análise, no qual o tema é apresentado; (ii) prática de discriminação, momento em que são aplicadas atividades de percepção; (iii) prática controlada e feedback, que consiste na repetição de termos propostos pelo professor, ainda que de forma mecânica; (iv) prática guiada e feedback, no qual os alunos utilizam as palavras do passo anterior de forma autônoma, sem interferência do professor; e (v) prática comunicativa e feedback, quando o aprendiz é capaz de desenvolver os aspectos trabalhados em um contexto sociocomunicativo orgânico.

Tendo o exposto como norte, este trabalho tem como objetivo principal investigar a realização e o desenvolvimento das consoantes oclusivas surdas iniciais do inglês-L2 (/p, t, k/) por falantes nativos do PB, soteropolitanos, estudantes de um curso de Letras-Inglês em uma universidade de Salvador/BA ${ }^{3}$. Tomamos como hipótese que estudantes de terceiro semestre, falantes independentes de língua inglesa (níveis B1 e B2 conforme ALLAN, 2004), de forma geral, não produziriam o padrão de VOT esperado para o inglês (long lag), transferindo e adotando, assim, o padrão do PB (short lag). Após a instrução, contudo, hipotetizamos que os participantes desenvolveriam um padrão de VOT mais longo para a 
L2, já que estudos como o de Sancier e Fowler (1997) e Kupske (2016) revelam que falantes nativos de PB são capazes de obter produções de forma estatisticamente similar aos nativos do inglês no que concerne aos valores de VOT para as oclusivas surdas do inglês.

Para testar essa hipótese, as falas de 16 participantes voluntários, divididos em dois grupos, foram gravadas e analisadas acusticamente. O primeiro grupo, experimental, recebeu instrução explícita sobre a produção do VOT no inglês, tendo como base o ensino comunicativo de pronúncia proposto por Celce-Murcia et al (1996) e Celce-Murcia et al (2010). O segundo grupo, controle, não recebeu instrução. Os dados foram coletados em três momentos: (1) pré-teste, coleta de dados anterior à instrução; (2) pós-teste imediato, logo após a instrução; e (3) pós-teste postergado, coletado três meses após o pós-teste anterior. O presente trabalho, portanto, busca descrever os efeitos de curto e médio prazo da instrução explícita de pronúncia inserida em um modelo comunicativo no desenvolvimento do padrão de VOT do inglês por falantes soteropolitanos. Para tanto, este trabalho está dividido em quatro seções. Na primeira, delinearemos nossa metodologia. Na segunda seção, apresentaremos a descrição e análise dos dados. Partimos, então, para a discussão geral dos dados e, encerrando este trabalho, nossas considerações finais.

\section{Metodologia}

\subsection{Participantes}

Inicialmente, 18 participantes foram selecionados (oito do sexo feminino e dez do sexo masculino), voluntários, maiores de idade, estudantes da graduação em Letras-Inglês em uma universidade da cidade de Salvador/BA, nascidos e criados na capital baiana. Esses participantes foram divididos em dois grupos: experimental e controle. Apenas falantes independentes do inglês, classificados como B1 e B2 (ALLAN, 2004) foram considerados. Durante o processo, dois participantes desistiram da pesquisa, que prosseguiu com um total de 16 participantes $(\mathrm{N}=16)$ : oito para o grupo experimental e oito para o controle. Todos os participantes preencheram um questionário básico e um termo de consentimento livre esclarecido.

\subsection{Materiais e Procedimentos}

\subsubsection{Palavras-Alvo}

A literatura (SCHWARTZHAUPT, 2013; KUPSKE, 2016) aponta que, além do número de sílabas e da taxa de elocução, a altura da vogal seguinte afeta a produção das oclusivas. Apenas vogais posteriores foram consideradas para limitar a variação do VOT. Dessa forma, baseando-se em Kupske (2016), utilizamos as seguintes palavras: 
Tabela 1: Palavras-alvo

\begin{tabular}{lllllll}
\hline Oclusiva & $/ \mathbf{p} /$ & & $/ \mathbf{t} /$ & & $/ \mathbf{k} /$ & \\
\hline Vogal & Alta & Baixa & Alta & Baixa & Alta & Baixa \\
Seguinte & Posterior & Posterior & Posterior & Posterior & Posterior & Posterior \\
\hline \multirow{3}{*}{ Alvo } & Poodle & Pop & Tool & Tod & Cool & Cob \\
& Poof & Posh & Toot & Top & Coop & Cod \\
& Pool & Pot & Tooth & Toss & Coot & Cop \\
\hline
\end{tabular}

Fonte: Elaborado pelos autores (2019)

Além das palavras-alvo presentes na Tabela 1, outros 18 itens distratores, todos monossílabos do inglês (e.g., Lap, Not, Rat, etc.), foram utilizados. Dessa forma, cada rodada de gravação continha 36 itens a serem gravados, sendo 18 alvos e 18 distratores. Cada participante gravou 36 alvos e 36 distratores. Estes últimos foram ignorados na análise.

\subsubsection{Procedimentos de coleta e instrução}

Toda as etapas da coleta de dados, pré-teste, pós-teste imediato e pós-teste postergado, foram conduzidas em língua inglesa, com instrumentos e instruções escritas e orais na mesma língua. Na fase de pré-teste, anterior à instrução, os participantes de ambos os grupos foram gravados individualmente em cabine acústica. Um microfone profissional Rode NT1 foi utilizado. A gravação foi feita por meio do software Adobe Audition 2.0, com uma taxa de amostragem de $44.100 \mathrm{~Hz}$, em um MacBook Pro, 2.3 GHz Intel Core i5 Dual-Core, com 8 GB de RAM. Todas as produções foram gravadas em estéreo e posteriormente convertidas para mono, para que não se perdesse nenhuma faixa de gravação.

Nas sessões de coleta, seguindo-se Kupske (2016), os participantes deveriam fazer a leitura da seguinte frase-veículo: "I would say" (palavra-alvo). Cada voluntário gravou todo o bloco de palavras duas vezes e poderia fazer uma pausa entre cada bloco. Em cada bloco e para cada participante, as palavras-alvo foram reordenadas, garantindo que nenhum bloco, de nenhum dos participantes, apresentasse o mesmo ordenamento de itens-alvo, atenuando efeitos de ordem e de borda. Os participantes poderiam repetir qualquer item quando julgassem necessário. Em caso de repetição, a última produção foi utilizada na análise de dados.

Após a coleta de dados de pré-teste, os integrantes do grupo experimental participaram de uma sessão de ensino comunicativo de pronúncia, baseada em Celce-Murcia et al (2010). A sessão teve duração de 120 minutos e contemplou os cinco passos do modelo proposto. A produção das oclusivas surdas iniciais do inglês foi o foco. É válido ressaltar que as palavras presentes nos instrumentos de coleta não foram utilizadas pelos pesquisadores nos materiais apresentados durante a sessão de ensino de pronúncia.

$\mathrm{Na}$ fase de pós-teste imediato, após a sessão de ensino, tanto o grupo experimental, que recebeu instrução sobre a produção das oclusivas do inglês-L2, quanto o grupo controle, sem instrução, gravaram novos dados. A mesma metodologia da 
pré-testagem foi adotada, mas com ordenamentos distintos das palavras-alvo e distratores. Três meses após a segunda coleta de dados, um pós-teste postergado (uma nova coleta, utilizando a mesma metodologia anterior) foi conduzido.

\subsubsection{Análises de VOT e estatística}

A análise acústica dos dados foi feita por meio do software Praat (BOERSMA; WEENINK, 2019), versão para Mac 6.0.07. Existem vários critérios que podem ser adotados para a medição do VOT; contudo, novamente, seguimos Kupske (2016). Para a extração do valor absoluto do VOT para as oclusivas surdas, a duração em milissegundos entre o burst e o primeiro pulso regular da vogal seguinte foi medida. Na presença de bursts múltiplos, comum na velar, por exemplo, a medição foi feita a partir do último burst da sequência. Tanto valores absolutos de VOT quanto relativos em relação à frase-veículo foram coletados. Como os resultados estatísticos foram os mesmos, optamos por apresentar apenas os dados absolutos.

Para nossa análise estatística, contamos com um total de 1.728 itens-alvo, sendo 864 para cada grupo (resultado da multiplicação de18 palavras por 8 participantes por 3 condições e por 2 repetições). Os dados foram tratados estatisticamente com o auxílio do programa IBM SPSS 26.0 (International Business Machine Statistical Package for Social Studies, Chicago, IL) para Mac. O valor de $p$ considerado foi menor ou igual a 0,05 .

\section{Descrição e análise de dados}

Nesta seção, iremos apresentar a descrição e análise dos dados das coletas obtidas nas fases de pré-teste, pós-teste imediato (depois do grupo experimental ter recebido instrução) e pós-teste postergado (três meses após o pós-teste imediato).

\subsection{Análise dos dados de pré-teste: o estado inicial}

De maneira geral, todo o grupo de participantes $(\mathrm{N}=16)$, grupos controle $(\mathrm{N}=8)$ e experimental $(\mathrm{N}=8)$, obteve as médias de duração de $23 \mathrm{~ms}$ com um desvio padrão (DP) de $9 \mathrm{~ms}, 33 \mathrm{~ms}(\mathrm{DP}=12)$ e $50 \mathrm{~ms}(\mathrm{DP}=13 \mathrm{~ms})$ para a produção do VOT das oclusivas surdas bilabial, alveolar e velar do inglês-L2, na fase de pré-teste, respectivamente. Em termos descritivos, para /p/ do inglês-L2, podemos perceber que os valores apresentados pelos participantes estão mais próximos dos $12 \mathrm{~ms}$ esperados para o PB (ISTRE, 1980) do que dos $55 \mathrm{~ms}$ (TORIBIO et at., 2005) esperados para o inglês. Para /t/, notamos uma média de duração entre os valores esperados para o PB e para a língua inglesa, $18 \mathrm{~ms}$ e 70 ms (KUPSKE, 2016), respectivamente, mas ainda próxima do padrão da L1. No caso de $/ \mathrm{k} /$, novamente, podemos perceber uma média de duração de VOT quase intermediária entre os valores esperados para o $\mathrm{PB}$ e a língua inglesa, $38 \mathrm{~ms}$ e 80 ms (KUPSKE, 2016), respectivamente. Dessa forma, em linhas gerais, todos os participantes desta pesquisa, antes da sessão de instrução para a produção das 
oclusivas do inglês, não produziam esses segmentos com os valores de VOT esperados e descritos na literatura para a L2.

Ao fazermos uma análise individual para cada grupo, separando os participantes do grupo controle e do experimental, obtivemos os seguintes dados para a produção das oclusivas, presente na Tabela 2.

Tabela 2: Médias de VOT para o inglês-L2 (pré-teste)

\begin{tabular}{lll}
\hline Oclusiva & Grupo Controle & Grupo Experimental \\
\hline$/ \mathbf{p} /$ & $22 \mathrm{~ms}(\mathrm{DP}=10 \mathrm{~ms})$ & $21 \mathrm{~ms}(\mathrm{DP}=8 \mathrm{~ms})$ \\
\hline$/ \mathbf{t} /$ & $33 \mathrm{~ms}(\mathrm{DP}=16 \mathrm{~ms})$ & $33 \mathrm{~ms}(\mathrm{DP}=14 \mathrm{~ms})$ \\
\hline$/ \mathbf{k} /$ & $47 \mathrm{~ms}(\mathrm{DP}=12 \mathrm{~ms})$ & $53 \mathrm{~ms}(\mathrm{DP}=13 \mathrm{~ms})$ \\
\hline
\end{tabular}

Fonte: Elaborado pelos autores (2019)

Podemos constatar que os valores de VOT para o grupo controle e para o grupo experimental, na pré-testagem, são próximos. Notamos que a maior diferença de médias encontra-se na produção da oclusiva velar, uma diferença de 6 ms. Nos demais casos, a diferença entre participantes-controle e experimentais é de aproximadamente $1 \mathrm{~ms}$. Além disso, como é de se esperar para a produção dos sons da fala por aprendizes de uma L2, os desvios-padrão são elevados, muitas vezes maiores do que a metade dos valores das médias obtidas pelos grupos, indicando, assim, uma grande variabilidade na produção das oclusivas do inglês-L2 por falantes soteropolitanos, em ambos os grupos.

Para que pudéssemos, efetivamente, confirmar a não existência de diferenças na produção de VOT do inglês entre os grupos controle e experimental no pré-teste, verificada a normalidade dos dados ${ }^{4}$, o Teste de T para Amostras Independentes foi aplicado. $\mathrm{O}$ teste revelou que não há diferenças estatisticamente significativas entre as produções do grupo controle e do grupo experimental para nenhuma das oclusivas do inglês analisadas $(\mathrm{p}>0,05)$. Para $/ \mathrm{p} /, t(14)=$ $0,143, \mathrm{p}>0,05$. Para $/ \mathrm{t} /, t(14)=0,024, \mathrm{p}>0,05$. Para $/ \mathrm{k} /, t(14)=-1,249, \mathrm{p}>$ 0,05 . Dessa forma, o grupo controle e o grupo experimental, antes da instrução acerca da produção das oclusivas no inglês-L2, possuíam valores de VOT estatisticamente similares.

\subsection{Análise dos dados de pós-teste imediato e pós-teste postergado: desenvolvimento imediato e de médio prazo}

De maneira geral, no pós-teste imediato e no pós-teste postergado, os participantes $(\mathrm{N}=16)$, controle $(\mathrm{N}=8)$ e experimental $(\mathrm{N}=8)$, obtiveram as médias de duração de VOT para o inglês-L2 conforme apresentado na Tabela 3. Nesta tabela, os valores de pré-teste e dos alvos da L2 são apresentados para simples comparação. 
Tabela 3: Médias de VOT para o inglês-L2 ( $\mathrm{N}=16)$

\begin{tabular}{lllll}
\hline Oclusiva & Pré-Teste & Pós-Teste Imediato & Pós-Teste Postergado & Alvo-L2 \\
\hline$/ \mathbf{p} /$ & $23 \mathrm{~ms}(\mathrm{DP}=9 \mathrm{~ms})$ & $57 \mathrm{~ms}(\mathrm{DP}=43 \mathrm{~ms})$ & $33 \mathrm{~ms}(\mathrm{DP}=15 \mathrm{~ms})$ & $55 \mathrm{~ms}$ \\
\hline$/ \mathbf{t} /$ & $33 \mathrm{~ms}(\mathrm{DP}=12 \mathrm{~ms})$ & $71 \mathrm{~ms}(\mathrm{DP}=45 \mathrm{~ms})$ & $44 \mathrm{~ms}(\mathrm{DP}=16 \mathrm{~ms})$ & $70 \mathrm{~ms}$ \\
\hline$/ \mathbf{k} /$ & $50 \mathrm{~ms}(\mathrm{DP}=13 \mathrm{~ms})$ & $91 \mathrm{~ms}(\mathrm{DP}=42 \mathrm{~ms})$ & $68 \mathrm{~ms}(\mathrm{DP}=14 \mathrm{~ms})$ & $80 \mathrm{~ms}$ \\
\hline
\end{tabular}

Fonte: Elaborado pelos autores (2019)

Em termos descritivos, do grupo como um todo, podemos perceber que os valores apresentados pelos participantes no pós-teste imediato estão próximos dos $55 \mathrm{~ms}, 70 \mathrm{~ms}$ e $80 \mathrm{~ms}$, esperados para /p/, /t/ e /k/ do inglês, respectivamente (TORIBIO et at., 2005). Identificamos, também, pelos valores altos de desvio padrão, que há uma alta variabilidade nos dados de pós-teste, isto é, que existem produções muito baixas e muito elevadas de VOT para o inglês. Após um aumento das médias e da variabilidade na segunda etapa de coleta, notamos uma diminuição desses valores no pós-teste postergado; todavia, mais elevados do que no pré-teste. Por outro lado, como veremos nas seções seguintes, quando separamos os dados em função dos grupos analisados, grupo controle (o qual não recebeu instrução sobre a produção do VOT) e grupo experimental (que recebeu instrução), percebemos um cenário bastante diferenciado.

\subsubsection{Análise dos dados do grupo controle}

Para a coleta de pós-teste imediato e pós-teste postergado, o grupo controle $(\mathrm{N}=8)$ obteve as seguintes médias de duração de VOT para o inglês-L2:

Tabela 4: Médias de VOT para o inglês-L2 do Grupo Controle ( $\mathrm{N}=8)$

\begin{tabular}{lllll}
\hline Oclusiva & Pré-Teste & Pós-Teste Imediato & Pós-Teste Postergado & Alvo-L2 \\
\hline$/ \mathbf{p} /$ & $22 \mathrm{~ms}(\mathrm{DP}=10 \mathrm{~ms})$ & $20 \mathrm{~ms}(\mathrm{DP}=5 \mathrm{~ms})$ & $21 \mathrm{~ms}(\mathrm{DP}=5 \mathrm{~ms})$ & $55 \mathrm{~ms}$ \\
\hline$/ \mathbf{t} /$ & $33 \mathrm{~ms}(\mathrm{DP}=16 \mathrm{~ms})$ & $33 \mathrm{~ms}(\mathrm{DP}=9 \mathrm{~ms})$ & $31 \mathrm{~ms}(\mathrm{DP}=7 \mathrm{~ms})$ & $70 \mathrm{~ms}$ \\
\hline$/ \mathbf{k} /$ & $47 \mathrm{~ms}(\mathrm{DP}=12 \mathrm{~ms})$ & $54 \mathrm{~ms}(\mathrm{DP}=5 \mathrm{~ms})$ & $56 \mathrm{~ms}(\mathrm{DP}=4 \mathrm{~ms})$ & $80 \mathrm{~ms}$ \\
\hline
\end{tabular}

Fonte: Elaborado pelos autores (2019)

A Tabela 4 revela que os valores de VOT nas diferentes testagens são similares, conforme o esperado, já que esse grupo não recebeu instrução. Dessa forma, os valores para as oclusivas da L2 do grupo ainda estão baseados em um padrão de VOT esperado para o PB. O Gráfico 1 apresenta os bloxpots para as produções do grupo controle para os três testes.

Como o Gráfico 1 demonstra, a produção dos valores de VOT para o grupo controle nos três momentos é similar. O mesmo pode ser percebido no Gráfico 2, para /t/, e Gráfico 3, para /k/. 
Gráfico 1: VOT para /p/ (grupo controle)

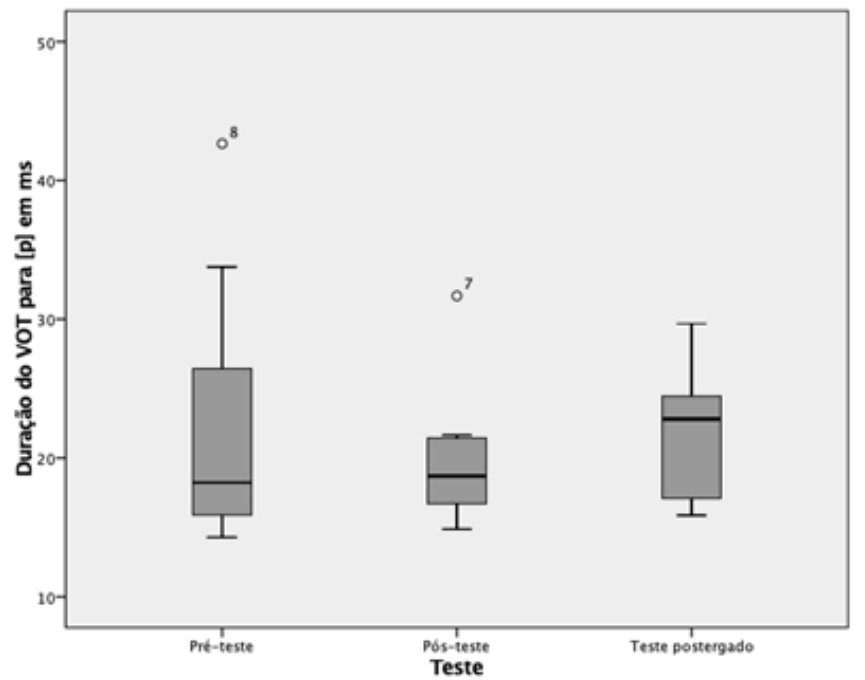

Fonte: Elaborado pelos autores (2019)

Como o Gráfico 1 demonstra, a produção dos valores de VOT para o grupo controle nos três momentos é similar. O mesmo pode ser percebido no Gráfico 2, para /t/, e Gráfico 3, para /k/.

Gráfico 2: VOT para /t/ (grupo controle)

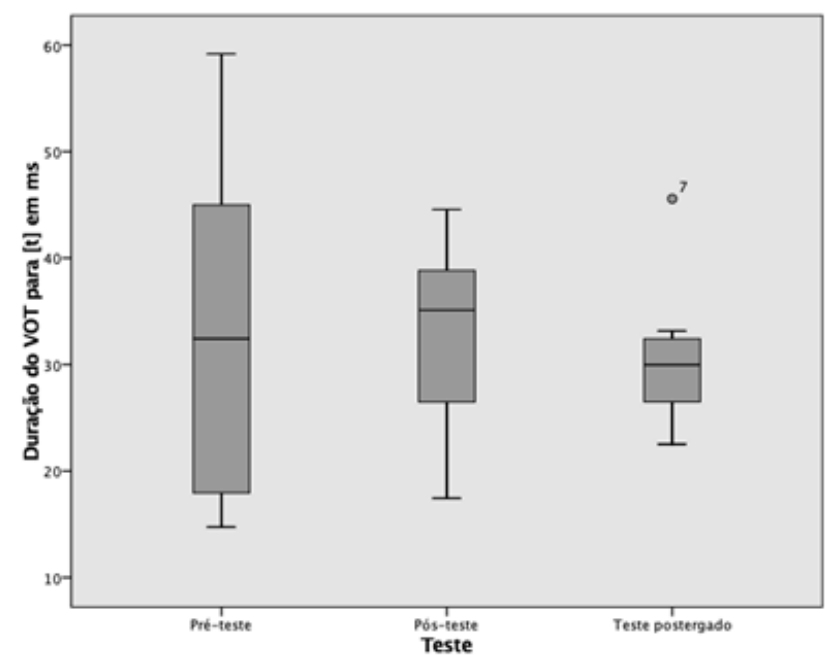

Fonte: Elaborado pelos autores (2019)
Gráfico 3: VOT para /k/ (grupo controle)

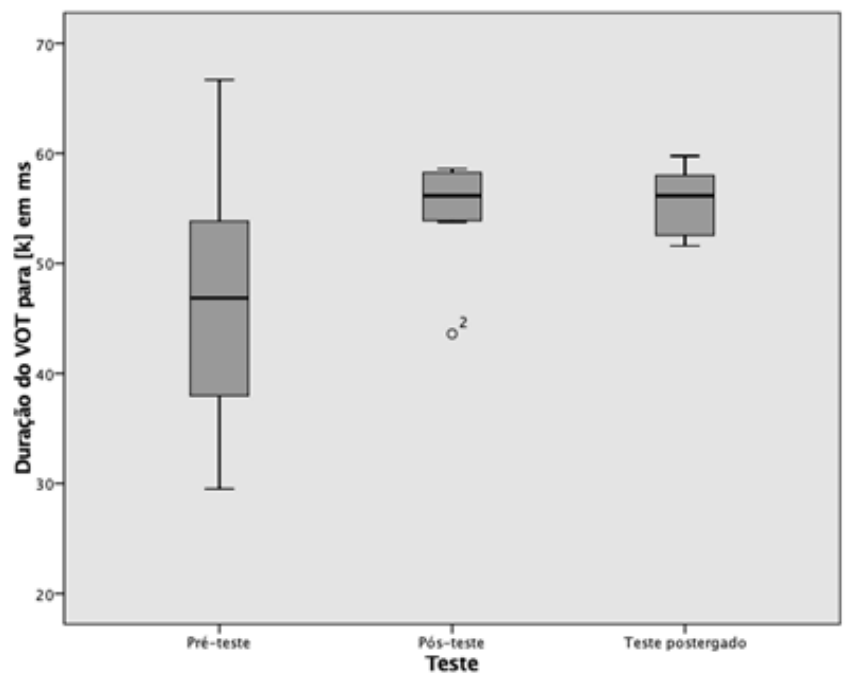

Como podemos inferir, em linhas gerais, os participantes do grupo controle não produzem esses segmentos com o padrão de VOT esperado para o inglês (long lag) em nenhum dos momentos de coleta, o que foi confirmado pela análise estatística. Sendo a distribuição dos dados normal e estando cumprido o Pressuposto da Esfericidade (Teste de Esfericidade de Mauchly), a Análise de Variância (ANOVA) para Medidas Repetidas revela que não há diferenças significativas entre os três momentos de coleta para a produção do VOT das oclusivas iniciais do 
inglês-L2 pelo grupo controle, $F(2,14)=10,981, \mathrm{p}>0,05, F(2,14)=13,454, \mathrm{p}>$ $0,05, F(2,14)=195,222, \mathrm{p}>0,05$, para a bilabial, alveolar e velar, respectivamente.

\subsubsection{Análise dos dados do grupo experimental}

Para a coleta de pós-teste imediato e pós-teste postergado (realizado três meses após a instrução), o grupo experimental $(\mathrm{N}=8)$ obteve as seguintes médias de duração de VOT para o inglês-L2:

Tabela 5: Médias de VOT para o inglês-L2 do grupo experimental $(\mathrm{N}=8)$

\begin{tabular}{lllll}
\hline Oclusiva & Pré-Teste & Pós-Teste Imediato & Pós-Teste Postergado & Alvo-L2 \\
\hline$/ \mathbf{p} /$ & $21 \mathrm{~ms}(\mathrm{DP}=8 \mathrm{~ms})$ & $93 \mathrm{~ms}(\mathrm{DP}=32 \mathrm{~ms})$ & $47 \mathrm{~ms}(\mathrm{DP}=15 \mathrm{~ms})$ & $55 \mathrm{~ms}$ \\
\hline$/ \mathbf{t} /$ & $33 \mathrm{~ms}(\mathrm{DP}=14 \mathrm{~ms})$ & $108 \mathrm{~ms}(\mathrm{DP}=33 \mathrm{~ms})$ & $54 \mathrm{~ms}(\mathrm{DP}=15 \mathrm{~ms})$ & $70 \mathrm{~ms}$ \\
\hline$/ \mathbf{k} /$ & $56 \mathrm{~ms}(\mathrm{DP}=13 \mathrm{~ms})$ & $127 \mathrm{~ms}(\mathrm{DP}=28 \mathrm{~ms})$ & $81 \mathrm{~ms}(\mathrm{DP}=11 \mathrm{~ms})$ & $80 \mathrm{~ms}$ \\
\hline
\end{tabular}

Em termos descritivos, para $/ \mathrm{p} /$, podemos perceber que a média de duração de VOT apresentada pelos membros do grupo experimental, no pós-teste imediato, é bastante superior (72 $\mathrm{ms}$ acima) à média de $21 \mathrm{~ms}$ produzida na prétestagem. Três meses após a instrução, a média para a bilabial é reduzida para 47 ms, número próximo aos $50 \mathrm{~ms}$ esperados para o inglês (TORIBIO et at., 2005). Para a oclusiva alveolar, a média para a segunda coleta é $75 \mathrm{~ms}$ maior quando comparada aos dados da coleta de pré-teste. Novamente, no pós-teste postergado, a média de /t/ representa um valor mais curto e próximo aos $70 \mathrm{~ms}$ esperados para o inglês (KUPSKE, 2016). Para a velar, assim como nos casos anteriores, a média de VOT no pós-teste imediato é superior à média esperada para a média do inglês, $80 \mathrm{~ms}$. No postergado, a média do grupo desce para $81 \mathrm{~ms}$, próxima ao padrão esperado para o inglês. Além das médias, devemos reparar nos desvios -padrão (DP) das testagens. O aumento dos DP na segunda coleta indica uma maior variabilidade na produção dos dados da L2. Por outro lado, a diminuição da variabilidade no pós-teste postergado revela um padrão mais consistente de produção para as oclusivas, com menor variabilidade, levando-nos a pensar em desenvolvimento do padrão de VOT para o inglês-L2, sobretudo, percentualmente, para a bilabial e para a velar. Os gráficos abaixo descrevem a produção do grupo nas diferentes testagens. 


\section{Gráfico 4: VOT para /p/ (grupo experimental)}

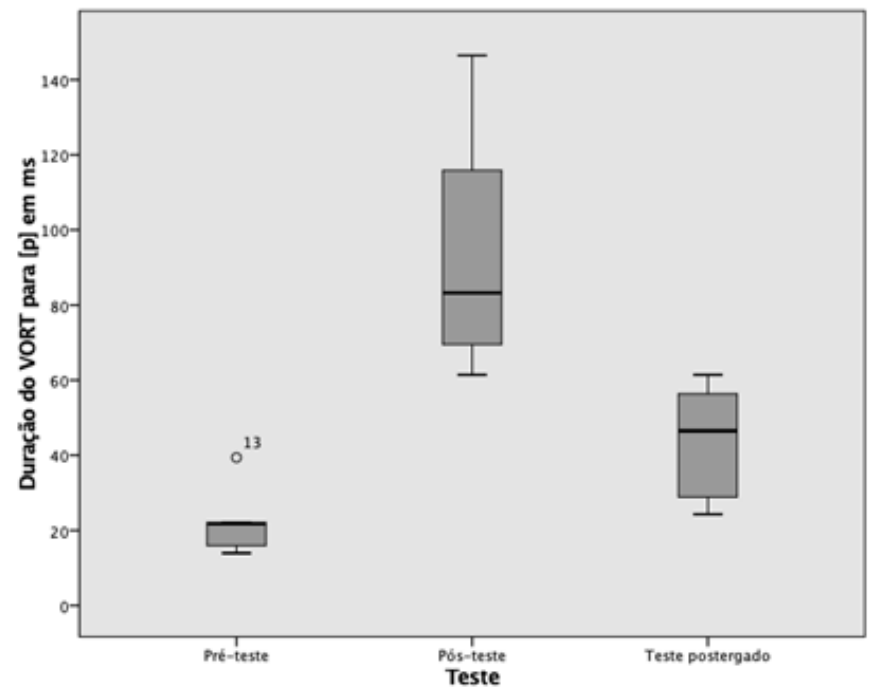

Fonte: Elaborado pelos autores (2019)

Gráfico 5: VOT para /t/ grupo experimental

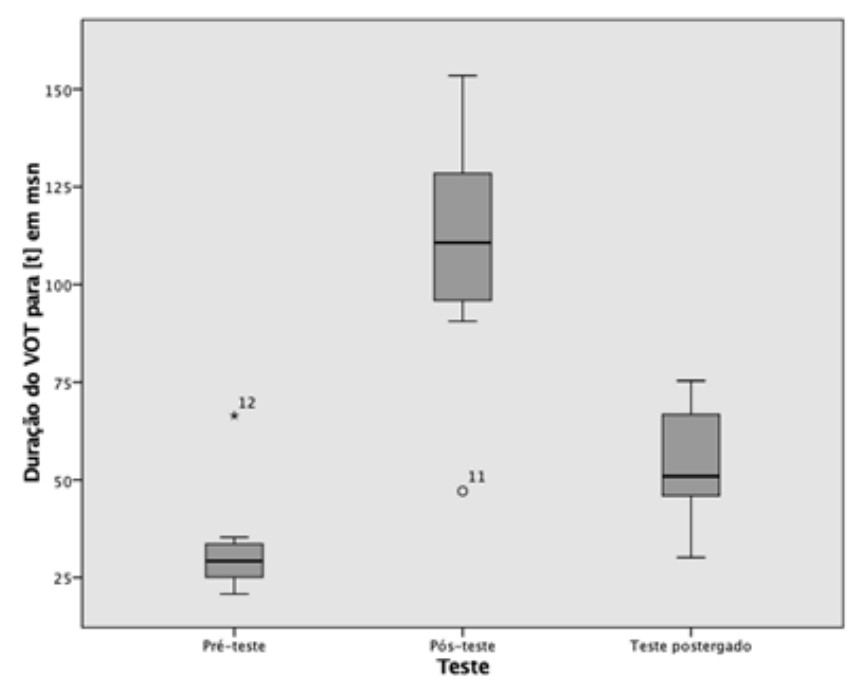

Gráfico 6: VOT para /k/ grupo experimental

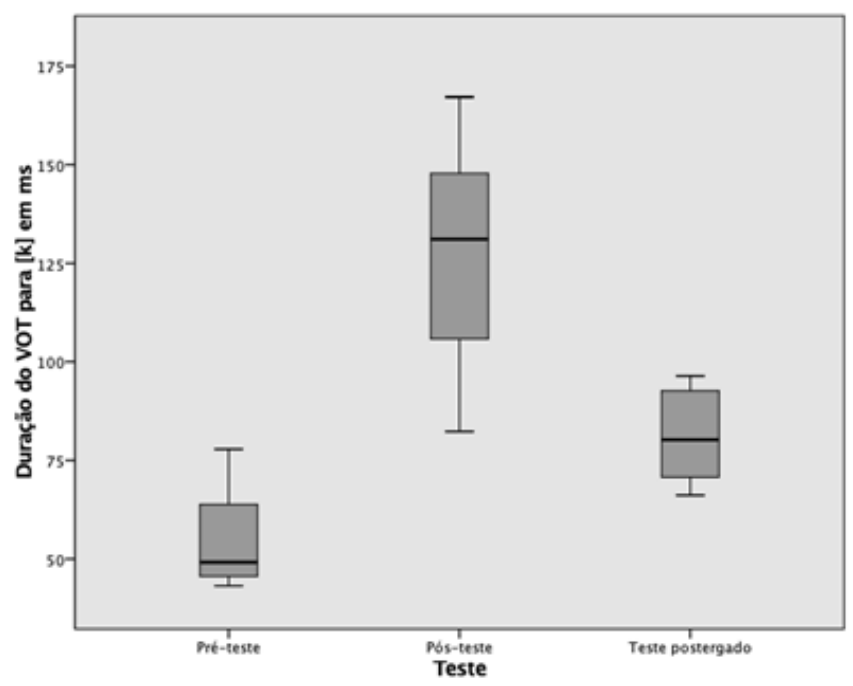

Fonte: Elaborado pelos autores (2019)

Em linhas gerais, os participantes do grupo experimental, logo após a sessão de instrução, produzem um VOT mais longo, aspirado. Contudo, apresentam valores muito acima daqueles esperados para o inglês. Após três meses, há uma redução nos valores de VOT e na variabilidade dos dados, mas, ainda assim, próximos daqueles esperados para a língua-alvo em comparação à primeira coleta, anterior à aplicação do modelo de Celce-Murcia et al (1996) e Celce-Murcia et al (2010). Sendo a distribuição dos dados normal e estando cumprido o Pressuposto da Esfericidade, a Análise de Variância (ANOVA) para Medidas Repetidas revela os dados a seguir.

Para a bilabial do inglês-L2, a ANOVA revela que há diferenças significativas entre as médias de VOT nas diferentes coletas, $F(2,14)=23,309, \mathrm{p}<0,001$. As comparações de efeitos principais de Bonferroni demonstram que as médias para 
/p/ no pós-teste imediato e no pós-teste postergado, mais elevadas, são diferentes da média do pré-teste $(\mathrm{p}<0,01)$. Contudo, as médias das duas últimas etapas de coleta também são estatisticamente diferentes entre si $(\mathrm{p}<0,05)$, sendo a média do pós-teste imediato, logo após a instrução, a mais elevada. Para /t/, a ANOVA revela que há diferenças significativas entre as médias de VOT nas diferentes coletas, $F(2,14)=24,238, \mathrm{p}<0,001$. As comparações de efeitos principais revelaram que a média de duração de / $\mathrm{t} /$ no pré-teste é estatisticamente diferente $(\mathrm{p}<0,01)$ da apresentada no pós-teste imediato, mas similar a do pós-teste postergado ( $\mathrm{p}>$ $0,05)$. As médias da alveolar no pós-teste imediato são estatisticamente diferentes da reveladas pelo pré-teste e pelo pós-teste postergado $(\mathrm{p}<0,01)$. Finalizando a análise estatística, a ANOVA apresenta diferenças significativas entre os momentos de coleta $F(2,14)=30,596, \mathrm{p}>0,001$ para a oclusiva velar. As comparações de efeitos principais de Bonferroni revelaram que há diferenças estatisticamente significativas entre todas as comparações possíveis $(\mathrm{p}<0,05)$.

\section{Discussão geral dos dados}

Conforme mencionado em nossa introdução, o VOT é uma importante pista acústica no inglês para o estabelecimento do contraste entre oclusivas iniciais surdas e sonoras. É importante, assim, para a inteligibilidade do inglês falado ao redor do mundo por falantes oriundos de diferentes contextos. A proposta de "pesos de pistas" (HOLT; LOTTO, 2006) advoga que as oposições entre segmentos podem variar em função das diversas pistas acústicas que os falantes de uma dada língua tomam como centrais. O VOT, por exemplo, parece ser a pista principal para a distinção entre oclusivas surdas e sonoras no inglês, mas não é uma pista utilizada por falantes do PB. Para Alves e Kampff (2019), embora não se saiba precisamente quais são as pistas utilizadas em PB para a distinção entre oclusivas surdas e sonoras, diversos estudos (ALVES; ZIMMER, 2015; SCHWARTZHAUPT; ALVES; FONTES, 2015) revelam que, ao contrário dos monolíngues do inglês, a presença ou ausência de VOT longos, aspiração, não é central para essa distinção. Assim, ao não possuir um papel principal na percepção dos sons da fala, o VOT "tampouco assumirá um status fundamental na produção de tais aprendizes. Trata-se de um fato importante, pois a não produção da aspiração em palavras tais como pit, tip e kill pode afetar a inteligibilidade da fala de tais aprendizes quando ouvida por ouvintes cuja L1 é o inglês" (ALVES; KAMPFF, 2019, p. 379).

Para Zimmer e Alves (2006), o comprometimento neuronal com a L1 resulta em transferência linguística. Uma das manifestações dessas transferências é o fato de que aprendizes apresentam mecanismos perceptuais entrincheirados na L1 e nem sempre eficientes em tarefas de percepção e produção de sons de uma L2 que, por exemplo, não contemplem ou contemplem sons muito próximos da língua materna. É por esse motivo que os participantes sem instrução, grupo controle, realizam um padrão de VOT esperado para o PB em seus alvos da L2. As oclusivas surdas em início de palavra do PB e do inglês poderiam ser consideradas, à luz de Flege (1995), como produções "similares". Dessa forma, as 
oclusivas surdas do inglês, mais longas (long lag), são percebidas por meio de categorias pré-existentes da L1, VOT mais curto (short lag), sendo, então, altamente suscetíveis a serem produzidas com este padrão. Para Best e Tyler (2007), os sons da L1 e L2 coexistem em um espaço fonológico único, oferecendo contexto para a transferência. Transferência, logo, é tida como a interação dos sistemas de L1 e L2 que coabitam nesse mesmo espaço fonológico (ALVES; SCHWARTZHAUPT; BARATZ, 2011). Nessa perspectiva, McKeough (1995) aponta que a transferência é uma mecanismo-chave que subjaz qualquer tipo de aprendizado por meio da aplicação do conhecimento já estabelecido a novas situações, como na L2. MacWhinney (2006) aponta que L1 e L2 estão representadas em uma única arquitetura neural, sendo a transferência, então, um fenômeno esperado como resultado da consolidação de mecanismos de memória, quantidade e qualidade de input. É nesse sentido, e à luz dos dados de nosso grupo controle, que concordamos com Alves e Kampff (2019) acerca da importância de práticas pedagógicas que guiem os aprendizes a adotarem o VOT como uma pista central para o inglês-L2 (ALVES; KAMPFF, 2019).

Carlet e de Kivistö-de Souza (2018) apontam que a pronúncia é um componente crucial para o desenvolvimento de uma L2, sendo dependente da exposição dos aprendizes a input de qualidade para ser aprimorada com sucesso (CELCE-MURCIA et al., 1996). No cenário de desenvolvimento no inglês-L2 no Brasil, onde aprendizes têm limitações em relação a oportunidades de interação com a L2, propostas que visem a melhoria da inteligibilidade são fundamentais. Nesse sentido, Gordon e Darcy (2016) indicam que o ensino explícito de pronúncia seria necessário para o desenvolvimento das habilidades orais de aprendizes de L2. Para Carlet e Kivistö-de Souza (2018), embora existam resultados desencorajadores sobre o efeito da instrução de pronúncia na produção de sons da L2, estudos mais recentes têm demonstrado efeitos positivos em relação aos efeitos da instrução de pronúncia na melhoria da percepção e/ou produção de sons L2, como no caso deste trabalho.

Com base na descrição e análise dos dados apresentados anteriormente, pudemos observar que a coleta de pré-teste apresenta valores similares de VOT para ambos os grupos, experimental e controle. Em termos gerais, percebemos que as médias de VOT das oclusivas surdas do inglês, para ambos os grupos, estariam distantes das esperadas para falantes do inglês, língua que utiliza o VOT como critério acústico distintivo. Percebemos que o grupo controle não produz aspiração na coleta de pré-teste e nem nas coletas de pós-teste imediato e pós-teste postergado, como esperado, e continuaram a transferir o padrão de VOT do PB para a L2. Por outro lado, na fase das coletas de pós-teste imediato, após os alunos do grupo experimental terem participado da sessão de instrução explícita, notase um aumento considerável de seus valores para as oclusivas da L2. Em termos gerais, os participantes utilizam a aspiração, mas não conseguem adequá-la aos valores apresentados pela literatura para o inglês. Em outras palavras, eles ainda não são capazes de controlar a produção do VOT, apresentando valores exagerados, muito embora já o apliquem nos contextos esperados. 
$\mathrm{Na}$ terceira etapa de coleta, tornamos a verificar, no grupo experimental, índices maiores de produção do VOT do inglês comparados aos valores do préteste. Contudo, no pós-teste postergado, os participantes apresentaram valores mais modalizados, não tão exagerados para a L2. Pensamos, assim, que houve uma melhora nos índices de produção do VOT, possibilitada pelo treinamento realizado e pelo aumento da consciência fonológica da L2 oriundo da adoção do modelo proposto por Celce-Murcia et al (1996) e Celce-Murcia et al (2010).

Estudos anteriores já indicaram que aprendizes de L2 que estão mais conscientes de sua pronúncia na língua-alvo e que possuem consciência das diferenças fonológicas entre a L1 e L2 apresentam uma produção mais acurada da língua-alvo (BAKER; TROIMOVICH, 2006). Schmidt (1995) aponta que o desenvolvimento da L2 não ocorre sem que o aprendiz perceba a L2. Assim, na área da oralidade da L2, é necessário que o aprendiz perceba os sons da L2 para o seu desenvolvimento. Defendemos, então, que a sessão de instrução de pronúncia contribuiu para que aprendizes do grupo experimental passassem a prestar mais atenção no VOT do inglês, fazendo, assim, com que ajustassem gradualmente a produção de VOT ao longo do tempo. Em outras palavras, os participantes começaram a controlar o VOT da L2. Assim, muito embora pesquisas anteriores sugiram que a quantidade e a qualidade de input podem não ser ideais no ambiente de sala de aula de L2 (DERWING; MUNRO, 2015), a aplicação do modelo revelou dados interessantes, sobretudo para a produção da oclusiva bilabial e velar da L2.

\section{Considerações finais}

Este trabalho tinha o objetivo principal de investigar a realização e o desenvolvimento das consoantes oclusivas surdas iniciais do inglês-L2 por aprendizes soteropolitanos, tomando a hipótese de que falantes independentes de língua inglesa não produziriam o padrão de VOT esperado para o inglês, adotando, assim, o padrão do PB. Após a instrução, contudo, a hipótese era de que os valores para a L2 iriam aumentar em direção ao long lag da L2. As duas hipóteses foram confirmadas, sendo que as médias de duração para as oclusivas estudadas nos dados de pós-teste postergado ficaram próximas às relatadas pela literatura para o inglês, sobretudo para as oclusivas bilabial e velar.

O grupo experimental que, no início do estudo, apresentava valores estatisticamente similares aos do grupo controle para as oclusivas do inglês, após a sessão de instrução, apresentou valores mais altos, mesmo que iniciando um alongamento de VOT de forma não finamente controlada, já que a mecânica gestual da aspiração é adquirida gradualmente. Assim, ao contrário de Saito (2012), que investigou os efeitos de um curto período de ensino de pronúncia explícita em L2 (quatro horas) para a produção de inglês-L2, este estudo revela que a instrução explícita foi capaz de introduzir a aspiração no grupo experimental, mesmo que ainda de forma não totalmente controlada.

Além de evidenciar o efeito da instrução explícita para o desenvolvimento do VOT do inglês-L2, nossos dados vão ao encontro de Sancier e Fowler (1997) e 
Kupske (2016, 2017), os quais afirmam que falantes de PB são capazes de atingir produções (estatisticamente) nativas do inglês, ao menos no que concerne ao VOT. Vão ao encontro, também, dos postulados de Flege (1995) e de Best e Tyler (2007), de que os mecanismos e processos de aprendizagem permanecem intactos na vida de um indivíduo, levando-nos a considerar que, de fato, tanto L1 e L2 coabitam um mesmo espaço no cérebro. Além disso, a regularização dos valores de VOT dos participantes do grupo experimental evidencia a hipótese de Flege (1995) e de Best e Tyler (2007) de que categorias fonéticas evoluem ao longo do tempo.

\section{Agradecimentos}

Agradecemos ao Conselho Nacional de Desenvolvimento Científico e Tecnológico (CNPq) por financiar o projeto de pesquisa (Processo n²32396/20187) ao qual este estudo está vinculado. Agradecemos, também, aos avaliadores anônimos pelos comentários e sugestões. Este estudo foi realizado no Laboratório de Ciências da Fala, LAFALA-UFBA.

Notas

1. É válido ressaltar que não descartamos outras pistas, além das acústicas, para a inteligibilidade na L2, como as presentes no próprio contexto de enunciação, tampouco afirmamos que a não aspiração acarretará, compulsoriamente, em ininteligibilidade. Contudo, isolamos os aspectos fônicos, nosso recorte.

2. Neste trabalho, utilizamos o termo L2 e LE de maneira intercambiável, como sinônimos de língua não nativa.

3. Este trabalho é parte de uma pesquisa maior e eticamente apreciada e aprovada. Certificado de Apresentação para Apreciação Ética: 52113615.0.0000.0116.

4. Para a análise da distribuição das variáveis intervalares, para que se verifique os princípios que subjazem à utilização de testes paramétricos, observamos os resultados exploratórios dos testes de normalidade Kolmogorov-Smirnov e Shapiro-Wilk, observando-se a normalidade da distribuição dos dados e a homogeneidade das variâncias por meio do Teste de Levene.

\section{Referências}

ALLAN, D. Oxford Placement Test 1. Oxford: Oxford University Press, 2004.

ALVES, U.; MAGRO, V. Raising awareness of L2 phonology: explicit instruction and the acquisition of aspirated /p/ by Brazilian Portuguese speakers. Letras de Hoje, Porto Alegre, v. 46, n. 3, p. 71-80, jul./set. 2011.

ALVES, U. K.; KAMPFF, F. Efeitos de longo prazo do treinamento perceptual na percepção e produção das plosivas iniciais surdas do inglês por estudantes brasileiros: implicações para o ensino de pronúncia. Itha do Desterro: A Journal of English Language, Literatures in English and Cultural Studies, Florianópolis, v. 72, n. 3, p. 375-400, 2019. Disponível em: https://doi.org/10.5007/21758026.2019v72n3p375.

ALVES, U.; SCHWARTZHAUPT, B.; BARATZ, A. Percepção e produção dos padrões de VOT do inglês (L2) por aprendizes brasileiros. In: FERREIRA-GONÇALVES, G.; BRUM-DE-PAULA, M. R.; KESKE-SOARES, M. (orgs.). Estudos em aquisição fonológica - Volume 4. Pelotas-RS: Editora e Gráfica Universitária - UFPel, 2011, p. $179-190$. 
ALVES, U. K.; ZIMMER, M. C. Percepção e produção dos padrões de VOT do inglês por aprendizes brasileiros: $\mathrm{O}$ papel de múltiplas pistas acústicas sob uma perspectiva dinâmica. Alfa: Revista de linguística, v. 59, p.155-175, 2015. Disponível em: https://doi.org/10.1590/1981-5794-1502-7.

BAKER, W.; TROIMOVICH, P. Perceptual paths to accurate production of L2 vowels: the role of individual differences. International Review of Applied Linguistics in Language Teaching, v. 44, p. 231-250, 2006. Disponível em: https:// doi.org/10.1515/IRAL.2006.010.

BECKER, M. Globalização, inglês como língua franca e inteligibilidade. In: BRAWERMAN-ALBINI, A.; GOMES, M. (org.). O jeitinho brasileiro de falar inglês. Campinas: Pontes, 2014. p. 223-239.

BEST, C.; TYLER, M. Nonnative and second-language speech perception: commonalities and complementarities. In: BOHN, O.; MUNRO, M.(org.). Language experience in second language speech learning: In honor of James Emil Flege. Amsterdam: John Benjamins, 2007. p. 13-34.

BOERSMA, P.; WEENINK, D. Praat: doing phonetics by computer. Versão 6.0.07, 2019. Disponível em: http://www.praat.org.

CARLET, A.; KIVISTÖ-DE SOUZA, H. Improving L2 pronunciation inside and outside the classroom. Ilha do Desterro: A Journal of English Language, Literatures in English and Cultural Studies, Florianópolis, v. 71, n. 3, p. 99-124, sep. 2018. Disponível em: https://doi.org/10.5007/2175-8026.2018v71n3p99.

CELCE-MURCIA, M. et al. Teaching pronunciation: a reference for teachers of English to speakers of other languages. Cambridge: Cambridge University Press, 1996.

CELCE-MURCIA, M. et al. Teaching pronunciation: a course book and reference guide. Cambridge: Cambridge University Press, 2010.

CRISTÓFARO-SILVA, T. et al. Fonética Acústica: os sons do português brasileiro. São Paulo: Editora Contexto, 2019.

DERWING, T. M.; MUNRO, M. J. Pronunciation fundamentals: evidence-based perspectives for L2 teaching and research. Netherlands: John Benjamins Publishing Company, 2015. (v. 42).

FLEGE, J. E. Second language speech learning: Theory, findings, and problems. In: STRANGE, W. (ed.). Speech perception and language experience: Issues in crosslanguage research. Baltimore: York Press, 1995, p. 233-277.

GORDON, J.; DARCY, I. The development of comprehensible speech in L2 learners: A classroom study on the effects of short-term pronunciation instruction. Journal of Second Language Pronunciation, vol. 2, n. 1, p. 56-92, 2016. DOI:10.1075/ jslp.2.1.03gor.

HOLT, L.; LOTTO, A. Cue weighting in auditory categorization: implications for first and second language acquisition. The Journal of the Acoustical Society of America, v. 119, n. 5, p. 3059-3071, 2006. Disponível em: https://doi.org/10.1121/1.2188377.

KUPSKE, F. F. Imigração, atrito e complexidade: a produção das oclusivas surdas iniciais do inglês e do português por brasileiros residentes em Londres. 2016. Tese (Doutorado em Letras) - Instituto de Letras, Universidade Federal do Rio Grande do Sul, Porto Alegre, 2016. 233 f.

KUPSKE, F. F. A complex approach on integrated late bilinguals' English VOT production: a study on South Brazilian immigrants in London. Ilha do Desterro: A Journal of English Language, Literatures in English and Cultural Studies, Florianópolis, v. 70, n 3, p. 81-93, set./dez. 2017. Disponível em: https://doi. org/10.5007/2175-8026.2017v70n3p81. 
KUPSKE, F. F.; ALVES, U. Orquestrando o caos: O ensino de pronúncia de língua estrangeira à luz do paradigma da complexidade. Fórum Linguístico, Florianópolis, v. 14, n. 4, p. 2771-2784, out./dez. 2017. Disponível em: https://doi. org/10.5007/1984-8412.2017v14n4p277.1

ISTRE, G. L. Um estudo do VOT em monolíngües brasileiros. Estudo não publicado. Universidade Federal de Santa Catarina, Florianópolis, Brazil., 1980.

JENKINS, J. The phonology of English as an international language: new models, new norms, new goals. Oxford, GB: Oxford University Press, 2000.

LISKER, L.; ABRAMSON, A. S. A cross-language study of voicing in initial stops: Acoustical measurements. Word, vol. 20, n. 3, p. 384-422, 1964.

MAURANEN, A.; RANTA, E. English as a lingua franca: Studies and findings. Cambridge: Cambridge Scholars Publishing, 2009.

MACWHINNEY, B. Emergentism: Use often and with care. Applied Linguistics, n. 27, p. 729-740, 2006. Disponível em: https://10.1093/applin/aml035.

McKEOUGH, A. Teaching for transfer: Fostering generalization in learning. Mahwah, NJ: Lawrence Erlbaum, 1995.

REIS, M. S.; NOBRE-OLIVEIRA, D. Effects of perceptual training on the identification and production of the English voiceless plosives aspiration by Brazilian EFL learners. In: NEW SOUNDS, 2007, Florianópolis. Proceedings of the Fifth International Symposium on the Acquisition of Second Language Speech. Florianópolis: UFSC, 2007. p. 398-407.

REIS, M. S.; NOBRE-OLIVEIRA, D.; RAUBER, A. Effects of perceptual training on the identification and production of the English voiceless plosives by Brazilian EFL learners. In: NEW SOUNDS, 2007, Florianópolis. Proceedings of the Fifth International Symposium on the Acquisition of Second Language Speech. Florianópolis: UFSC, 2007. p. 25-28.

SAITO, K. Effects of instruction on L2 pronunciation development: A synthesis of 15 quasi-experimental intervention studies. TESOL Quarterly, v. 46, n. 4, p. 842-854, 2012.

SANCIER. M. L.; FOWLER, C. A. Gestural drift in a bilingual speaker of Brazilian Portuguese and English. Journal of Phonetics, v. 27, n. 4, p. 421-436, 1997.

SCHMIDT, R. Consciousness and foreign language learning: a tutorial on the role of attention and awareness in learning. In: SCHMIDT, R. (ed.). Attention and awareness. Honolulu, HI: University of Hawaii, National Foreign Language Resource Center, 1995.

SCHWARTZHAUPT, B. M. Factors influencing Voice Onset Time: analyzing Brazilian Portuguese, English and interlanguage data. 2013. Trabalho de Conclusão de Curso - Instituto de Letras, Universidade Federal do Rio Grande do Sul, Porto Alegre, 2013.

SCHWARTZHAUPT, B. M.; ALVES, U. K.; FONTES, A. B. A. L. The role of L1 knowledge on L2 speech perception: investigating how native speakers and Brazilian learners categorize different VOT patterns in English. Revista de Estudos da Linguagem, v. 23, n. 2, p. 311-334, 2015.

SEIDLHOFER, B. Understanding English as a Lingua Franca. Oxford: Oxford University Press, 2011.

SILVEIRA, R.; ALVES, U. Noticing e instrução explícita: Aprendizagem fonéticofonológica do morfema-ed. Nonada Letras em Revista, vol. 2, n. 13, p. 149-159, 2009. 
THOMSON, R.; DERWING, T. The effectiveness of L2 pronunciation instruction: A narrative review. Applied Linguistics, vol. 36, n. 3, p. 326-344, 2015. DOI: https://10.1093/ applin/amu076.

TORIBIO, A; BULLOCK, B.; BOTERO, C.; DAVIES, K. Perseverative phonetic effects in bilingual code-switching. In: GESS, R. S.; RUBIN, E. J. (eds.). Theoretical and experimental approaches to Romance linguistics. Salt Lake City: John Benjamins, 2005, p. 291-306.

ZIMMER, M.; ALVES, U. A aprendizagem de L2 como processo cognitivo: a interação entre conhecimento explícito e implícito. Nonada, Porto Alegre, v. 9, p. 157-174, 2006.

Recebido em: 30/03/2020

Aceito em: 30/06/2020 
